Archives

18-19 | 1997

Inventaire des archives de Robert Mandrou

\title{
Le 27 juin 1995
}

Christiane Mandrou

\section{(2) OpenEdition \\ Journals}

\section{Édition électronique}

URL : http://journals.openedition.org/ccrh/2555

DOI : $10.4000 /$ ccrh. 2555

ISSN : $1760-7906$

Éditeur

Centre de recherches historiques - EHESS

Édition imprimée

Date de publication : 10 octobre 1997

ISSN : 0990-9141

Référence électronique

Christiane Mandrou, «Le 27 juin 1995 », Les Cahiers du Centre de Recherches Historiques [En ligne], 18-19 | 1997, mis en ligne le 20 février 2009, consulté le 20 avril 2019. URL : http:// journals.openedition.org/ccrh/2555; DOI : 10.4000/ccrh.2555

Ce document a été généré automatiquement le 20 avril 2019

Article L.111-1 du Code de la propriété intellectuelle. 


\title{
Le 27 juin 1995
}

\author{
Christiane Mandrou
}

1 Je m'étais rendue, sans grand enthousiasme, je l'avoue, à la discrète cérémonie du 27 juin 1995 qui devait marquer la cession des papiers personnels de mon mari, le professeur Robert Mandrou, aux Archives nationales, par l'intermédiaire du Centre de recherches historiques de l'École des hautes études en sciences sociales, manifestation tout à fait représentative, par sa modestie et son atmosphère chaleureuse, des rapports qu'entretenait l'École avec Robert Mandrou. Et un doute persistant, quant au bien-fondé de ma décision, subsistait en moi, en dépit des nombreux échanges téléphoniques et épistolaires que j'avais eus avec Lucette Valensi et Brigitte Mazon.

2 Puisque l'œuvre de Robert Mandrou était dans une large mesure plus appréciée et reconnue à l'étranger que dans son propre pays - c'est à l'ambassade du Canada à Paris qu'a eu lieu, le 28 janvier 1986, l'unique cérémonie officielle à sa mémoire -, il m'avait paru évident que le seul endroit pour accueillir ces admirables notes de cours et de conférences, cette correspondance riche de plusieurs milliers de lettres qui tissait à travers les continents, des liens entre des chercheurs déjà prestigieux ou dont la reconnaissance était encore à venir, l'endroit où mon mari aurait été heureux de voir ses archives accueillies était la Mission historique française en Allemagne, à Göttingen. Pendant de nombreuses années, soutenu par Carlo Schmid et les historiens allemands, notamment le professeur Rudolf Vierhaus, directeur du Max-Planck Institut pour l'histoire à Göttingen, qui voyaient en lui le grand historien français indépendant qu'il était, il avait mobilisé tout son prestige, tout le jeu de ses relations amicales - je pense en particulier à Michel Debré, dont l'intervention a été déterminante pour faire sortir le projet des cartons du Quai d'Orsay où il sommeillait depuis fort longtemps - et ses forces déclinantes, face au mal qui le rongeait, pour mettre sur pied cet institut de recherches historiques franco-allemand dont il rêvait. Mais il n'a pu aller au-delà de la fondation et c'est à Étienne François, qu'il avait souhaité voir lui succéder à la tête de la Mission historique française en Allemagne, qu'en échurent l'organisation et la mise en route concrètes. C'est ce même Étienne François, sur la fidèle amitié duquel je savais pouvoir compter, qui, après mûre réflexion, m'a conseillé d'opter finalement pour la proposition 
du Centre de recherches historique de l'École des hautes études en sciences sociales, assurant la conservation et la consultation des papiers de Robert Mandrou, par la pérennité institutionnelle des Archives nationales.

3 Cette longue incidente pour réaffirmer que c'est à Étienne François que le Centre de recherches historique doit finalement ma décision; et pour faire comprendre aussi que l'agacement qu'ont fait naître en moi certaines publications mal informées ou outrageusement subjectives aurait pu me conduire à l'annuler. Mais les compétences, l'intelligence et le tact des archivistes de l'École des hautes études en sciences sociales, Brigitte Mazon et Marie-Annick Morisson, m'ont alors rassurée sur mon choix. Depuis un an et demi que nous travaillons ensemble à rassembler et classer toutes les traces écrites de l'activité de Robert Mandrou, je me félicite d'un engagement qui m'a permis de mettre de l'ordre dans mes souvenirs autant que dans les dossiers, pour magistralement classés qu'ils fussent déjà, de mon mari ; dossiers qui, au terme d'un inventaire - ici publié sous forme sommaire-, permettent de réaliser combien lumineux et rigoureux était son esprit.

4 Cette plongée quasi quotidienne dans les archives personnelles de Robert Mandrou m'a fait revivre nos vacances studieuses consacrées aux archives françaises lors de nos petits congés scolaires, ou aux archives allemandes pendant les mois d'été. De Rennes à Besançon, de Bordeaux à Carpentras, d'Auch à Troyes, par exemple, quelle diversité entre les dépôts et leurs responsables... Mais pour nous, les horaires étaient toujours les mêmes -9 heures-12 heures -14 heures-18 heures. Robert dépouillait et je rédigeais les fiches lorsque le texte à conserver avait une certaine longueur. Ces fiches sont toujours là, nettes et précises jusque dans leur classement. Pourtant les scrupules de Robert Mandrou, son honnêteté intellectuelle - un modèle dans un milieu où l'on devrait pourtant être à l'abri des généralisations hâtives - le conduisaient parfois à ne pas se fier uniquement à ses notes : j'ai gardé dans ma mémoire, en partie à cause de son inconfort, ce long été pluvieux et froid, dans le grenier des archives des Fugger à Dilligen-an-der-Donau - amicalement prêté par Maria Gräfin von Preysing, l'archiviste - et consacré à la rédaction de la petite thèse... Ainsi était-il à même de retourner aux sources en cas d'hésitation. Pour nous, vacances et archives étaient confondues ; mais c'est le travail de l'historien, sa raison d'être, et la visite des musées s'inscrivait seulement dans la partie récréative de nos séjours, quel que fût le rôle qu'ils jouaient dans l'enrichissement de nos connaissances. Les vacances de remise en forme ne se sont que tardivement imposées à nous : elles consistaient en longues promenades tout terrain, motivées par la cueillette des champignons, que j'ai appris aussi à identifier grâce à lui, qui tenait sa science de son séjour forcé du service du travail obligatoire (STO), effectué dans le Harz ; de mon côté, je l'initiais aux joies des découvertes minéralogiques.

5 Le STO a joué un rôle déterminant dans les relations de Robert Mandrou avec l'Allemagne et l'Europe centrale et orientale, et mes propres origines familiales maternelles ont renforcé cet attachement à une culture, à des paysages qui nous ont apporté beaucoup de joies et d'amitiés dont je garde précieusement le souvenir. Je me souviens des difficultés à l'accepter d'une de nos amies les plus chères, Etty Wodowski-Menahem, dont nous avions fait la connaissance à Tel-Aviv où elle enseignait le français à l'université; notre relation privilégiée avec l'Allemagne la heurtait, elle dont les parents avaient tragiquement disparu pendant ces sombres années où l'Europe baignait dans l'horreur nazie. Elle nous aimait trop, cependant, pour ne pas l'accepter, mais son incompréhension est restée totale jusqu'à sa mort. 
6 C'était cette faculté rare, possédée par Robert Mandrou, de susciter sympathie et attachement chez la plupart de ceux qu'il rencontrait, de quelque catégorie sociale qu'ils fussent, qui me paraît le trait le plus admirable, le plus caractéristique de sa personnalité. Il le devait à sa simplicité, à son intérêt sincère pour les hommes qui le mettait naturellement à l'écoute de tous; et ceux qui le côtoyaient le ressentaient immédiatement, de ses compagnons d'atelier à la Hermann-Goering-Stahlwerke de Wattenstedt aux étudiants de Paris X (il n'aimait guère que d'autres que lui disent Nanterre), en passant par les Américains qui lui confièrent, à leur arrivée, la responsabilité des travailleurs étrangers à Seesen... Il vivait pleinement ce que Marc Bloch a affirmé dans son Apologie pour l'histoire :

«[...] ce sont les hommes que l'histoire veut saisir. Qui n'y parvient pas ne sera jamais, au mieux, qu'un manœuvre de l'érudition ».

7 Car cet homme si intimidant au milieu de ses pairs, était pour tous les autres d'un abord étonnamment facile. Une anecdote illustrera parfaitement ce que je viens de dire. Lors de l'unique visite que nous fîmes à un quelconque Salon de l'automobile, où nous devions retrouver des amis, nous nous vîmes offrir, dans le métro, par un inconnu, dont le fils était exposant, des billets d'entrée, simplement parce qu'il nous avait écoutés deviser et qu'il avait jugé que nous étions un « couple rare »... Le charme de Robert avait opéré, tout comme il a opéré sur l'infirmière qui m'a secondée pendant les derniers mois, et qui a senti pourtant ce qu'avait d'exceptionnel sa personnalité, et l'horreur d'une telle maladie pour un homme tel que lui. Tous les 25 mars depuis treize ans, elle dépose discrètement, sur l'appui de ma fenêtre, les tulipes rouges qu'il lui avait dit préférer à toute autre fleur... Mais c'est ce même Robert Mandrou qui répondit, brièvement, du haut de son podium, à un ambassadeur en poste, qui s'excusait de devoir partir avant la fin de son exposé :

« tant pis pour vous, monsieur l'Ambassadeur».

Je ne doute pas que ce soit ce chaleureux intérêt porté aux autres qui a rassemblé, en ce mois de juin 1995, autant d'amis de Robert Mandrou, collègues ou administratifs de l'École, dont la présence et les interventions m'ont si profondément émue, et que j'ai le sentiment d'avoir si mal remerciés de leur fidélité à sa mémoire et à son amitié. Je tiens à ce qu'ils sachent combien j'ai eu de bonheur à les entendre, même s'ils évoquaient des souvenirs qui restent, et resteront, toujours aussi douloureux pour moi. Si j'ai avoué avoir détesté l'École ou plutôt le séminaire animé par Robert, c'est parce que j'étais jalouse de ceux qui profitaient sans moi de son intelligence aiguë. Il n'aurait tenu qu'à moi, pourtant, de ne pas enseigner le samedi matin et de l'accompagner. Mais je préférais le laisser libre d'éprouver ses idées, ses hypothèses, au contact de cerveaux brillants, dont certains sont devenus des amis, évoqués toujours avec tendresse - une tendresse retenue, certes, mais très perceptible-dans nos conversations: Jean-Paul Aron, lui aussi tragiquement disparu; Gérard Bouchard, qui, pendant un certain temps, passait le prendre à l'appartement avant de rejoindre la « rue de Varenne »; Françoise Parent, la seule à l'appeler " patron »; Jean-Pierre Peter... Cohorte de fidèles à laquelle se sont joints, en d'autres lieux et en d'autres temps, Jean Lecuir, Monique et Bernard Cottret, Philippe Joutard, Étienne François enfin, qui œuvrent toujours à la sauvegarde de sa mémoire. Le temps des déjeuners à La Petite Chaise, qui suivaient le séminaire, des stages en province qui clôturaient l'année, est passé. Nous sommes maintenant dans celui de la conservation du souvenir, des souvenirs devrais-je dire, pour que d'autres que nous, qui l'avons tant aimé et admiré, soient fascinés par la lumineuse simplicité de son intelligence, et se posent avec moi la question qui me hante: pourquoi ce gâchis? 
Pourquoi cette carrière si tôt brisée, alors qu'il avait encore tant à nous apporter, qu'il allait enfin pouvoir, libéré de la lourde charge de son enseignement à Paris $\mathrm{X}$, se consacrer aux projets qui lui tenaient à cœur, qui auraient ouvert la voie de nouvelles recherches à la communauté des historiens et nous aurait donné, entre autres choses, cette étude sur Port-Royal et ce récit consacré à notre histoire, qu'il rêvait d'écrire? Hasard ou nécessité? 